\title{
Microwave Phase Contrast Imaging of the Subsurface using Variation in Soil Moisture Level
}

\author{
Sebastian G. Wirth*, Ivor L. Morrow*, Daniel Andre* \\ ${ }^{*}$ Cranfield University, Laboratory of Electromagnetic Systems Engineering, \\ Shrivenham Campus, Oxfordshire, SN6 8LA, UK. \\ Email: sebastian.wirth@ cranfield.ac.uk
}

\begin{abstract}
A new microwave subsurface imaging product is described which is evolved via time lapsed microwave measurements, over several days, of the soil subsurface. The technique exploits changes in soil moisture levels that occur naturally due to evaporative and percolation processes. A novel technique is investigated for detecting and discriminating buried targets; it provides a scaled phase weighting procedure to form a cumulative B-scan image of the subsurface. The algorithm is demonstrated on ground penetrating radar measurements acquired in the $X$ Band spectrum over soil where reference targets and other buried artefacts are placed. Early stage experimental results suggest the approach shows promise to provide enhanced subsurface imagery with reduced clutter and noise levels.
\end{abstract}

\section{INTRODUCTION}

Ground based and near-ground penetrating radar systems are the only practical method for high resolution and subsurface imaging. These systems rely on detailed knowledge of the monostatic, bistatic or multi-static antenna properties and geometric configuration to spatially isolate vertically the subsurface and surface backscatter return signals. Cross range resolution can, of course, be improved using synthetic aperture processing (SAR) [1]; that again requires a priori knowledge of the measurement setup. A novel microwave imaging technique is described that offers alternative subsurface imaging capabilities. The advantage is, it does not rely upon a knowledge of the measurement setup or using noise and clutter suppression techniques [2]. The principle mechanism exploits naturally occurring changes in soil moisture level to affect a change in the dielectric properties of the subsurface medium. Isolating changes in electrical phase of subsurface features, from those that do not, presents the possibility to effectively separate buried targets from the background scene. A disadvantage of the scheme is the requirement for repeated measurement over relatively long periods of time.

The measurement of soil moisture levels with radar has been a subject of long standing interest [3], [4], [5] as has the determination of the relative permittivity of soil-water mixtures at microwaves frequencies [6]. More recently researchers have reported the use of soil moisture change to provide virtual bandwidth synthetic aperture radar imagery [7]. Underpinning these techniques is the need for a soil model that adequately predicts the changes in complex relative permittivity and observed effects at frequencies from 1.4-20 GHz. The presented technique is aimed to address a capability gap of imaging and detecting landmines in the subsurface [8].
This paper is organised as follows: Section II presents the elements of a model formulation that accounts for the observed effects of various soil components on the complex dielectric behaviour of the soil-water-air system as a function of frequency. In Section III the laboratory experimental setup and methodology are described. Section IV presents the results of analysis using these new subsurface imaging technique. Section $\mathrm{V}$ provides a critical engineering assessment of the technique.

\section{SOIL MODEL}

\section{A. Four Component Dielectric Mixing}

The Dobson [6] dielectric mixing model is generally acknowledged as a reliable predictor of the dielectric behaviour of wet soil. The model is developed from appropriate empirical and theoretical dielectric mixing models and requires only volumetric moisture and soil textural composition as inputs.

A four component dielectric mixing model treats the soilwater system as a host medium of dry soil containing randomly distributed disc shaped inclusions of bound water, bulk water and air. The relative permittivity of the mixture $\dot{\epsilon}_{m}$ can be written [6].

$$
\dot{\epsilon}_{m}=\frac{3 \epsilon_{s}+2 V_{f w}\left(\dot{\epsilon}_{f w}-\epsilon_{s}\right)+2 V_{b w}\left(\epsilon_{b w}-\epsilon_{s}\right)+2 V_{a}\left(\epsilon_{a}-\epsilon_{s}\right)}{3+V_{f w}\left(\epsilon_{s} / \dot{\epsilon}_{f w}-1\right)+V_{b w}\left(\epsilon_{s} / \epsilon_{b w}-1\right)+V_{a}\left(\epsilon_{s} / \epsilon_{a}-1\right)}
$$

where the subscripts $b w, f w, a$, and $s$ refer to bound water, free water, air and soil. Volume fractions for each component are calculated for each soil at a given volume water content and bulk density of the soil; $\epsilon_{a}=1.0004$ and $\epsilon_{s}$ is determined from the empirical fit to the measured data presented in [10]. $\dot{\epsilon}_{f w}$ is derived from a Debye type relaxation that accounts for ionic conductivity losses as,

$$
\epsilon_{f w}^{\prime}=\epsilon_{w \infty}+\frac{\epsilon_{w 0}-\epsilon_{w \infty}}{1+\left(2 \pi f \tau_{w}\right)^{2}}
$$

and

$$
\epsilon_{f w}^{\prime \prime}=\frac{2 \pi f \tau_{w}\left(\epsilon_{w \infty}-\epsilon_{w \infty}\right)}{1+\left(2 \pi f \tau_{w}\right)^{2}}+\frac{\sigma_{m_{v}}}{2 \pi \epsilon_{0} f} .
$$

$\epsilon_{w 0}$ is the static dielectric constant of water, $\epsilon_{w \infty}=4.49$ is the high frequency limit and $\epsilon_{0}$ has its normal value. $\sigma_{m_{v}}$ is the effective conductivity of the volume of bound water and $\tau_{w}$ is the relaxation time of pure water and given by, 


$$
\begin{array}{r}
2 \pi \tau_{w}(T)=1.1109 \times 10^{-10}-3.824 \times 10^{-12} T \\
+6.938 \times 10^{-14} T^{2}-5.096 \times 10^{-16} T^{3}
\end{array}
$$

where $T$ is in ${ }^{\circ} \mathrm{C}$. This expression was obtained by Stogyrn [9] by polynomial fitting to experimental data. An equivalent term cited is the relaxation frequency $f_{o}=\left(2 \pi \tau_{w}\right)^{-1}$, which for water in the microwave region $f_{0}\left(0^{\circ} C\right) \approx 8.9 \mathrm{GHz}$ and $f_{0}\left(20^{\circ} \mathrm{C}\right) \approx 16.7 \mathrm{GHz}$.

The complex relative permittivity, $\dot{\epsilon}_{m}$, is calculated using (1)-(4) over the $1.4-20 \mathrm{GHz}$ range of validity for the model. At a nominal frequency of $10 \mathrm{GHz}$ the relative permittivity of dry sand with a dry density of $1.6 \mathrm{~g} / \mathrm{cm}^{3}$ and volumetric water content of $0 \%$ at a laboratory temperature of $21.5^{\circ} \mathrm{C}$ is $\dot{\epsilon}_{m}(d r y)=(3.031-j 0)$. Fig. 1 plots the relative permittivity as a function of frequency for a $5 \%$ volumetric water content, which at $10 \mathrm{GHz}$ is estimated as $\dot{\epsilon}_{m}(w e t)=(7.0+j 0.75)$.

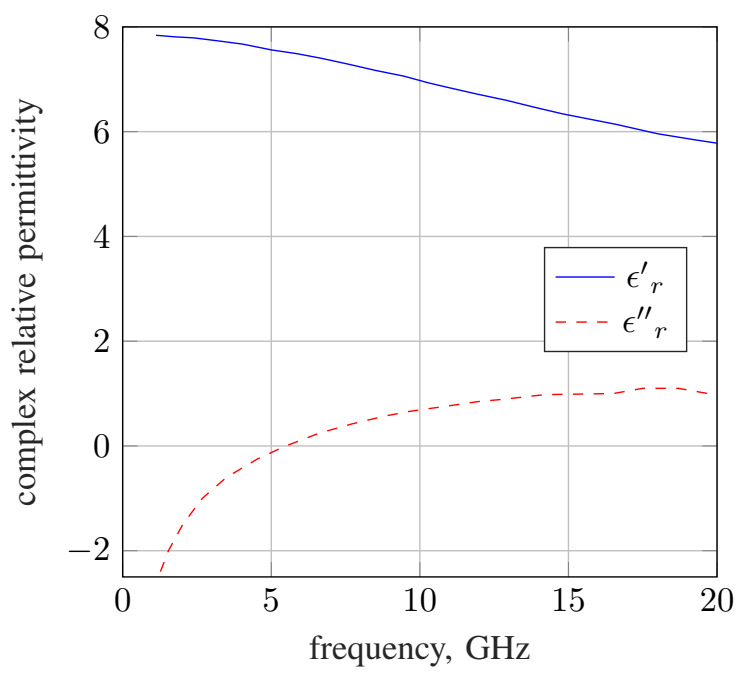

Fig. 1: Computed relative complex dielectric constant of $5 \%$ wet sandy soil.

\section{LABORATORY EXPERIMENT}

\section{A. Setup}

The microwave measurement facility consisted of a sand bay $3 \mathrm{~m}(\mathrm{l}) \times 1.2 \mathrm{~m}(\mathrm{w}) \times 0.8 \mathrm{~m}(\mathrm{~d})$. A $3.0 \mathrm{~m}$ linear actuator was located centrally along the length of the bay and used to scan four pyramidal horn antennas arranged in a polarimetry array. The antenna array is mounted $0.58 \mathrm{~m}$ above the soil surface and a Vector Network Analyser (VNA) used to collect X-Band (8.0-12.0 GHz) S-parameter measurements. Measurements are collected in $1.0 \mathrm{~cm}$ increments along the length of the scan and both actuator and VNA are under computer control. Measurements of VV, VH, HH, HV polarisations are made, using a bandwidth of $4.0 \mathrm{GHz}$. Fig. 2 shows a view of the measurement setup. The sand bay was filled with kiln dried $100 \%$ by volume sand and the surface was levelled. To control and monitor the sand wetting procedure two Perspex trays with the inner dimensions $0.7 \mathrm{~m} \mathrm{(l)} \times 0.7 \mathrm{~m}(\mathrm{w}) \times 0.07 \mathrm{~m}(\mathrm{~h})$ were constructed and placed on the surface of the sand bay. The moisture was restricted to the trays alone.

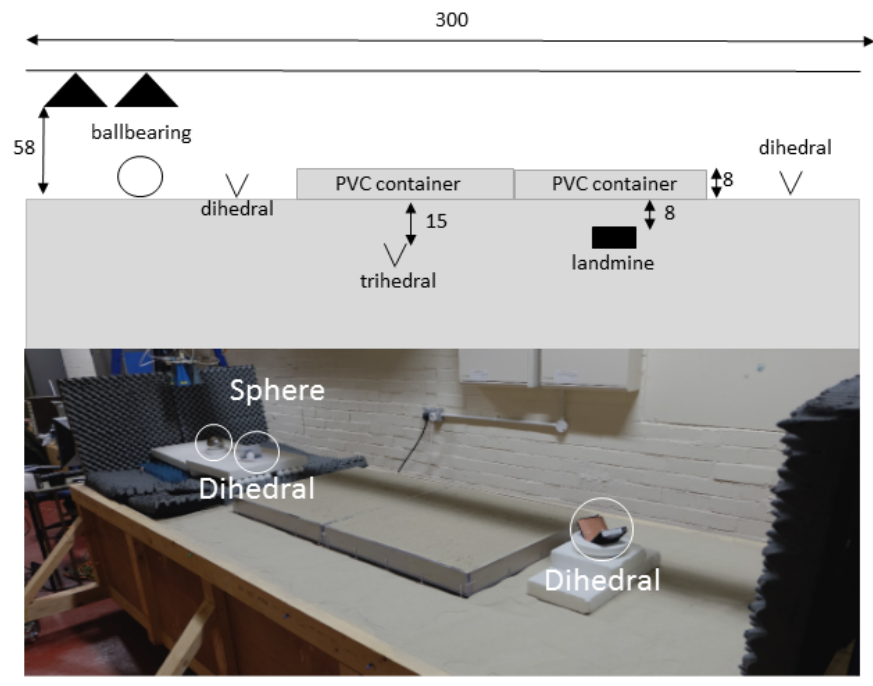

Fig. 2: Schematic diagram and photo showing a view of the experimental radar scene (units in $\mathrm{cm}$ ).

Several reference targets were placed above the surface of the sand on a bed of radar absorbent material (RAM) or support on polystyrene foam. These include a $40 \mathrm{~mm}$ metal sphere and two $70 \mathrm{~mm}$ square sided dihedral reflectors. Under the first Perspex tray a trihedral of $70 \mathrm{~mm}$ square sided was buried at a depth of $230 \mathrm{~mm}$ from the surface of the filled tray. Under the second Perspex tray a TS-50 landmine of diameter of $60 \mathrm{~mm}$ and thickness $40 \mathrm{~mm}$ was buried at a depth of $160 \mathrm{~mm}$ from the surface of the filled tray to the top of the landmine. The Perspex has a thickness of $10 \mathrm{~mm}$, thus the outer depth of the tray is $80 \mathrm{~mm}$. The sphere, dihedral and trihedral allow for GPR channel amplitude and phase correction.

\section{B. Methodology}

An initial control microwave scan and B-scan image was carried out with the soil dry.

The wetting phase:

- Deionised water in 3\% by volume of the tray (or equivalently $0.35 \mathrm{~L}$ ) was incrementally added to the Perspex tray.

- Three titrations were applied in one hour intervals.

- A Delta moisture meter was used to take reading of moisture after each titration.

- A measurement B-scan was then taken of the radar scene.

Following immediately on the drying phase:

- Every three hours a microwave B-scan measurement was made of the radar scene.

- Everyday a Delta moisture meter was used to take a reading of moisture level and the temperature was recorded.

- Microwave measurements were continued for 5-days and a total of 53 scans were acquired. 
When soil moisture levels in the Perspex trays had fallen to near zero the experiment was stopped. This collected data formed the basis of the analysis.

\section{ANALYSIS}

\section{A. Signal Processing}

The complex $S$-parameter measurements are converted to range profiles. The VNA uses a Stepped Frequency Continuous Waveform (SFCW) consisting of $N$ pulses, equally separated by $\Delta f$ Hertz. The phase of the sampled quadrature mixer output is,

$$
\phi_{n}=-2 \pi\left(f_{o}+n \Delta f\right) \frac{2 z}{c}
$$

where $z$ is the range to the object and $f_{o}$ is the frequency of the first transmitted pulse [11] may be expressed as the complex spectral return $S$ of amplitude $A_{n}$ as,

$$
S\left(f_{n}\right)=A_{n} \exp \left(j \phi_{n}\right) .
$$

The $N$ complex samples in each burst are Inverse Fast Fourier Transformed (IFFT) to a series of complex range reflectivity profiles. It can be shown that taking the IFFT (or FFT) of $M$ samples (from $N$ pulses) corresponding to a range bin divides the range bin into finer subdivisions resulting in a smoother range profile [12]. In this experiment a total of $N=1601$ sample steps were taken in each measurement and resampled to $M=4096$.

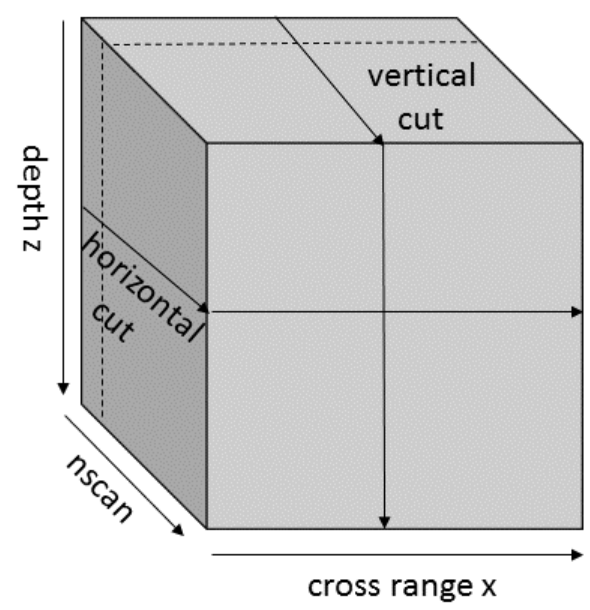

Fig. 3: Structure of data stored in each polarimetry data cube.

It is convenient to collect all the range profiles into a data cube as shown in Fig. 3. The cube holds the down-range $(z)$, the cross range scan position $(x)$ and the number of time lapsed rescans (nscan) for one polarisation. A total of four data cubes are required to represent all the polarisations measured. A typical B-scan image contained in the data cube is shown in Fig. 4. The surface laid and buried targets are all clearly visible.

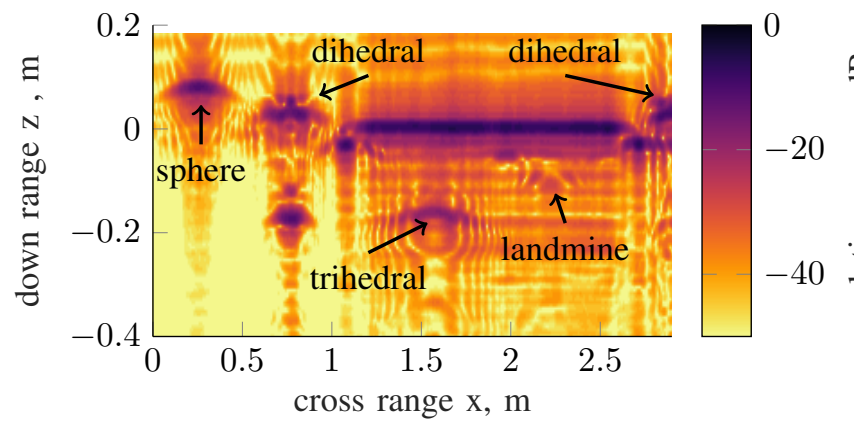

Fig. 4: B-scan image of the radar scene with the surface and buried targets indicated. (Note that downrange $0 \mathrm{~m}$ is referenced to the surface of the sand bay, not the top of the trays.)

\section{B. Moisture Change Detection}

A simple expression for predicting of the total phase change due to the change in relative permittivity $\dot{\epsilon}$ over the time lapsed series of collected B-scans images may be given as.

$$
\Delta \Phi=\frac{4 \pi}{\lambda} D\left(\sqrt{\left|\epsilon_{m}(w e t)\right|}-\sqrt{\left|\epsilon_{m}(d r y)\right|}\right)
$$

with $D$ being the depth of the target, $\epsilon_{m}$ (wet) the ("wet") relative dielectric constant and $\epsilon_{m}(d r y)$ the ("dry") dielectric constant. Substituting the values calculated from the Dobson model at $10.0 \mathrm{GHz}$, plotted in Fig. 1, corresponds to a predicted phase change of $383.75^{\circ}$ electrical degrees. Since the moisture level was not allowed to rise above 3\%, the Dobson model's dataset only allows a quantitative prediction of the absolute phase change with frequency. If the moisture level is allowed to rise above $3 \%$, up to a maximum of $60 \%$, then the Dobson model would allow prediction of phase change over a range of permittivities at a fixed frequency.

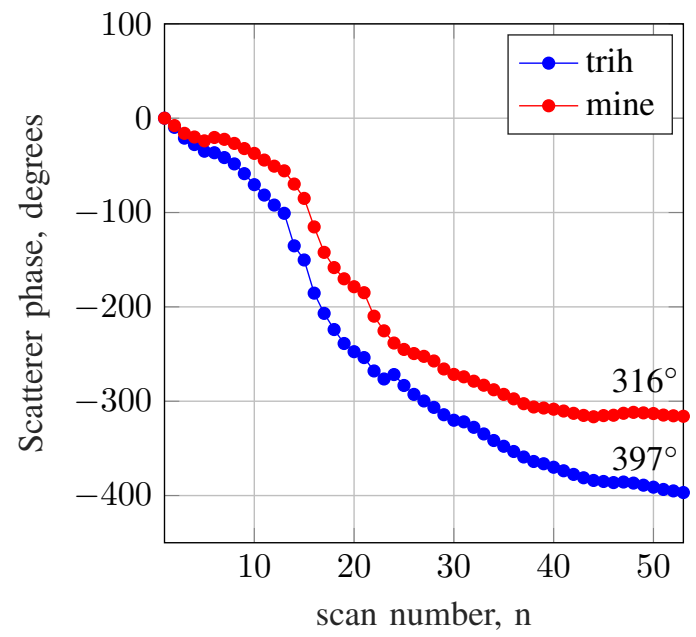

Fig. 5: Observed experimental phase change of the trihedral and TS-50 landmine over all time lapsed scans.

Experimentally, the phase change can be extracted from the datacube by monitoring the electrical phase in the B-scan at 


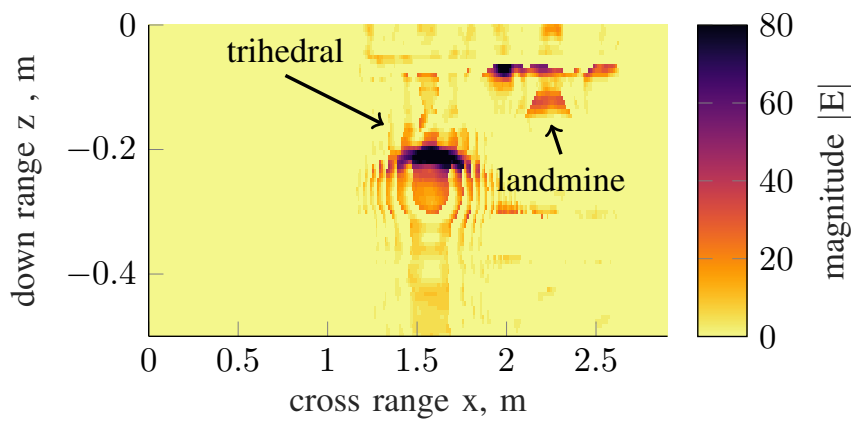

Fig. 6: Summed magnitude weighted differential phase contrast B-scan $(\alpha=1)$.

the pixel cells for the trihedral and TS-50 landmine. Fig 5 plots the phase history of these pixels over all the time lapsed scans. A measured phase change of $397^{\circ}$ over the trihedral and $316^{\circ}$ over the TS-50 landmine was observed and these results are in close agreement with our soil model prediction. If we take a Fast Fourier transform in the vertical or horizontal cut of the data cube, depicted in Fig 3, at the location of either the trihedral or the TS-50 landmine the resulting range profile demonstrates that the objects appear shifted down range by one range bin and is due to the relative permittivity change in the medium.

\section{Phase Weighted Imagery}

It is evident from Fig 4 that significant clutter is present in the subsurface radar scene. A novel technique was postulated to provide image clutter suppression. It was observed from plots of B-scan phase imagery that the phase changed only around buried objects as the soil dried and this was anticipated. An image based algorithm was formulated around this hypothesis and is given as;

$$
P(x, y)=\sum_{n=1}^{n s c a n} \Delta \phi(x, y)_{n} \cdot\left|p(x, y)_{n}\right|^{\alpha}
$$

where,

$$
\Delta \phi(x, y)_{n}=\angle\left(\frac{p(x, y)_{n+1}}{\left|p(x, y)_{n+1}\right|}\right) \cdot \frac{\left|p(x, y)_{n}\right|}{p(x, y)_{n}}
$$

where the summed $P(x, y)$ pixel is the magnitude weighted differential phase contrast of the complex valued $p(x, y)$ pixel at position $(x, y)$ of the individual $\mathrm{B}$-scan that compose the $n$ scans. The exponent $\alpha$ is either 1 for magnitude or 2 for intensity of the electric field, depending on the image format required.

The resultant image is shown in Fig 6 and it can be seen that stationary phase artefacts are mostly removed from the scene and those that change phase in a constant direction and have a scatterer present are retained. Hence the air-surface interface is almost completely suppressed and mostly only the buried targets, the trihedral and TS-50 landmine, are retained in the image.

\section{DISCUSSION}

This prediction and experimental study has demonstrated the potential to use natural changes in soil moisture levels to evolve new subsurface imaging products. A laboratory setup where moisture levels could be monitored and controlled while microwave measurements of the surface laid and buried targets were carried out. A complex soil model was validated and may be used to calibrate phase changes in the relative permittivity as the soil moisture level is reduced. It was shown that measured and calculated phase change are in agreement as in both cases the buried target experiences a range shift and appears in the next range bin down. A new approach for subsurface detection and imaging has been demonstrated. In particular the complex imaging algorithm can be used to significantly reduce surface and subsurface clutter compared to that produced using conventional GPR clutter suppress techniques. The use of this technique in field campaigns will likely throw up additional real-world application issues. However, the inclusion of a robust and comprehensive soil model, such as the Dobson used here, provides specific knowledge on the soil moisture level necessary to provide accurate phase change and depth scale.

\section{ACKNOWLEDGMENT}

The authors thank the Find A Better Way Charity for their support of this research under the DETERMINE programme (grant number 2015/001D).

\section{REFERENCES}

[1] C.V. Jackowatz et al., Spotlight-Mode Synthetic Aperture Radar: A Signal Processing Approach, Springer, 1996, ISBN 0-7923-9677-4.

[2] D.J. Daniels, Ground Penetrating Radar - 2nd Edition, IET, 2004, ISBN $0-86341-360-9$

[3] A. Benedetto, F. Benedetto Remote Sensing of Soil Moisture Content by GPR Signal Processing in the Frequency Domain, Antennas and Propagation (APSURSI), IEEE Sensors Journal, Vol. 11, No. 10, Oct. 2011.

[4] R Rajesh Mohan et al. Microwave imaging for soil moisture content estimation, IEEE International Symposium on Antennas and Propagation (APSURSI), 2016.

[5] J.E. Hipp, Soil electromagnetic parameters as functions of frequency, soil density, and soil moisture, Proceedings of the IEEE, 1974.

[6] M.C. Dobson, F. T. Ulaby, M.T. Hallikainen, M. A. El-Rayes, Microwave Dielectric Behaviour of Wet Soil Part II: Dielectric Mixing Models, IEEE Transactions on Geoscience and Remote Sensing, Vol. GE-23, No. 1, IEEE, 1985.

[7] A. Edwards-Smith and K. Morrison and S. Zwieback and I. Hajnsek Verification of the Virtual Bandwidth SAR Scheme for Centimetric Resolution Subsurface Imaging From Space, IEEE Transactions on Geoscience and Remote Sensing, Vol. 56, Issue 1, IEEE, Jan. 2018.

[8] R. van Dam, B. Borchers, J.M.H Hendricks Strength of landmine signatures under different soil conditions: implication for sensor fusion, International Journal of Systems Science, 2005, pp. 1-15.

[9] A. Stogyrn, Equations for Calculating the Dielectric Constant of Saline Water, IEEE Transactions on Microwave Theory and Techniques, Vol. 19, No. 8, pp. 733-736.

[10] M. T. Hallikainen; F. T. Ulaby; M. C. Dobson; M. A. El-rayes; L. Wu, Microwave Dielectric Behaviour of Wet Soil Part I: Empirical Models and Experimental Observations from 1.4 to $18.0 \mathrm{GHz}$, IEEE Transactions on Geoscience and Remote Sensing, Vol. GE-23, No. 1, IEEE, 1985.

[11] M. Skolnik, Radar Handbook - 2nd Edition, McGraw-Hill, 1990, ISBN 0-07-057913-X.

[12] D. R. Wehner. High Resolution Radar. Artect House, Chap. 5, second edition, 1995 